\title{
Manipulation of Majorana bound states in proximity to a quantum ring with Rashba coupling
}

Fabían Gonzalo Medina ( $\nabla$ fmmedinacfabian@gmail.com )

Universidad T' ecnica Federico Santa María

Dunkan Martínez

GISC, Universidad Complutense

Alvaro Díaz-Fernández

GISC, Universidad PolitécnicaPolit'Politécnica de Madrid

Francisco Domínguez-Adame

GISC, Universidad Complutense

Luis Rosales

Universidad T' ecnica Federico Santa María

Pedro A. Orellana

Universidad T' ecnica Federico Santa María

\section{Research Article}

Keywords: magnetic flux, theoretical proposals, physics. Majorana zero modes (MZM)

Posted Date: July 23rd, 2021

DOl: https://doi.org/10.21203/rs.3.rs-738291/v1

License: (c) (i) This work is licensed under a Creative Commons Attribution 4.0 International License.

Read Full License

Version of Record: A version of this preprint was published at Scientific Reports on January 20th, 2022. See the published version at https://doi.org/10.1038/s41598-022-05043-y. 


\title{
Manipulation of Majorana bound states in proximity to a quantum ring with Rashba coupling
}

\author{
Fabián Gonzalo Medina ${ }^{1,+, *}$, Dunkan Martínez ${ }^{2,+}$, Álvaro Díaz-Fernández ${ }^{3}$, Francisco \\ Domínguez-Adame ${ }^{2}$, Luis Rosales ${ }^{1}$, and Pedro A. Orellana ${ }^{1}$
}

\author{
${ }^{1}$ Departamento de Física, Universidad Técnica Federico Santa María, Casilla 110 V, Valparaíso, Chile \\ ${ }^{2}$ GISC, Departamento de Física de Materiales, Universidad Complutense, E-28040 Madrid, Spain \\ ${ }^{3}$ GISC, Departamento de Estructuras y Física de Edificación, Universidad Politécnica de Madrid, E-28031 Madrid, \\ Spain \\ *fmmedinacfabian@gmail.com \\ +These authors contributed equally to this work
}

\begin{abstract}
The quest for Majorana zero modes in the laboratory is an active field of research in condensed matter physics. In this regard, there have been many theoretical proposals; however, their experimental detection remains elusive. In this article, we present a realistic setting by considering a quantum ring with Rashba spin-orbit coupling and threaded by a magnetic flux, in contact with a topological superconducting nanowire. We focus on spin-polarized persistent currents to assess the existence of Majorana zero modes. We find that the Rashba spin-orbit coupling allows for tuning the position of the zero modes and has sizable effects on spin-polarized persistent currents. Our results pave the way towards probing the existence of Majorana zero modes.
\end{abstract}

\section{Introduction}

Majorana fermions remain a theoretical construct in the realm of particle physics. These particles, whose main feature is to be their own antiparticles, have been sought after in neutrinos, although without success thus far ${ }^{1,2}$. In condensed matter physics, Majorana quasiparticles emerge in $p$-wave topological superconductors and great progress has been made during the last few years in their experimental detection ${ }^{3,4}$. Of particular relevance are the so-called Majorana zero modes (MZM) ${ }^{2}$, which are zero-energy Majorana quasiparticles protected by particle-hole symmetry. These quasiparticles, in contrast to their particle counterparts, exhibit yet another crucial feature. Such property is their being non-Abelian under braiding, which renders these quasiparticles as ideal candidates to perform topological quantum computing ${ }^{5,6}$. The reason for this stems in the large degeneracy found in the ground state of a system comprising a large number of Majorana quasiparticles. This degeneracy occurs due to these zero modes being pinned to zero energy ${ }^{4}$.

The archetypal model for studying Majorana modes in superconductors is that of Kitaev ${ }^{7-9}$, where a one-dimensional spinless $p$-wave superconductor is considered. Under suitable tuning of parameters, the model predicts that an ordinary superconductor undergoes a topological quantum phase transition into a topological superconductor. When considering open boundary conditions, the model predicts an odd number of MZMs at both ends of the chain with exponential decay into the bulk. Kitaev's model, although simple, poses significant challenges for its experimental realization. However, a great deal of experimental advances have taken place during the last few years, and a convenient setting has been put forward ${ }^{4,10-12}$. Such a setting has three key ingredients: a semiconductor nanowire with large spin-orbit coupling, a uniform magnetic field parallel to the wire to induce Zeeman splitting, and an s-wave superconductor. When placed in contact with the semiconductor and applying the magnetic field, the superconductor becomes topological when a given magnetic field is reached.

\section{System and model Hamiltonian}

The Hamiltonian of the system is given by

$$
H=H_{r}+H_{M}+H_{c},
$$

where $H_{r}$ is the Hamiltonian of the ring, $H_{M}$ describes the interaction between the two MZMs at the edges of the nanowire, and $H_{c}$ contains the coupling between the MZMs and the quantum ring. Considering spin-orbit coupling and the fact that the 
quantum ring is threaded by a flux $\Phi, H_{r}$ can be written as ${ }^{13-15}$

$$
H_{r}=-\sum_{n}\left[\boldsymbol{c}_{n}^{\dagger}\left(\boldsymbol{t}+\boldsymbol{t}_{\mathrm{so}}(n)\right) e^{\mathrm{i} \theta} \boldsymbol{c}_{n+1}+\text { h.c. }\right]
$$

where $\boldsymbol{c}_{n}^{\dagger}=\left(c_{n, \uparrow}^{\dagger}, c_{n, \downarrow}^{\dagger}\right)$ are the creation operators of the quantum ring and $n$ runs from 1 to $N$, with $N$ the number of sites of the ring. We shall measure energies in units of $2 t$ with $t$ the hopping energy, which implies that $t=\sigma_{0} / 2$, with $\sigma_{0}$ the $2 \times 2$ identity matrix. The flux is accounted for in the phase factor $\theta=2 \pi \Phi / N$, where $\Phi=\phi / \phi_{0}$ is the flux $\phi$ measured in units of the flux quantum $\phi_{0}=h / e$. The spin-orbit coupling is given by

$$
\boldsymbol{t}_{\mathrm{so}}(n)=\mathrm{i} \tilde{\alpha}\left(\sigma_{x} \cos \varphi_{n}+\sigma_{y} \sin \varphi_{n}\right),
$$

where we only consider Rashba spin-orbit interaction. Here, $\sigma_{x}$ and $\sigma_{y}$ are the Pauli matrices, $\tilde{\alpha}=\alpha N / 2 \pi R$ being $R$ the radius of the ring, $\alpha$ the strength of the Rashba interaction and

$$
\varphi_{n}=\frac{2 \pi}{N}\left(n-\frac{1}{2}\right) \text {. }
$$

The interaction term between the two MZMs is given by ${ }^{7}$

$$
H_{M}=\mathrm{i} \xi_{M} \gamma_{1} \gamma_{2}
$$

where $\gamma_{1}$ and $\gamma_{2}$ are Majorana operators which satisfy $\gamma_{i}=\gamma_{i}^{\dagger}$ and $\left\{\gamma_{i}, \gamma_{j}\right\}=\delta_{i j}$. Here $\xi_{M} \propto e^{-L / \ell_{0}}$ where $L$ is the length of the nanowire and $\ell_{0}$ is the superconducting coherence length ${ }^{7}$. The Majorana operators can also be written in terms of ordinary fermion operators, $f$ and $f^{\dagger}$, as

$$
\gamma_{1}=\frac{1}{\sqrt{2}}\left(f+f^{\dagger}\right), \quad \gamma_{2}=\frac{\mathrm{i}}{\sqrt{2}}\left(f-f^{\dagger}\right) .
$$

In terms of these operators, Eq. (5) takes the form

$$
H_{M}=\xi_{M}\left(f^{\dagger} f-\frac{1}{2}\right)
$$

Finally, the coupling between the MZMs and the quantum ring is given by ${ }^{16-18}$

$$
H_{c}=\lambda_{1}\left(c_{1, \uparrow}^{\dagger}-c_{1, \uparrow}\right) \gamma_{1}+\mathrm{i} \lambda_{2}\left(c_{1, \uparrow}^{\dagger}+c_{1, \uparrow}\right) \gamma_{2},
$$

where $\lambda_{1}$ and $\lambda_{2}$ are real parameters. We have chosen to couple the MZMs to a single spin. This can be achieved by fixing the spin canting angles of the MZMs accordingly, which can be done by means of sufficiently large magnetic fields ${ }^{17}$.

It is now convenient to turn to a representation where $H_{r}$ is diagonal. For that matter, we can use the following unitary transformation ${ }^{14}$

$$
\mathscr{U}_{n}=\frac{1}{\sqrt{2}}\left(\begin{array}{cc}
1 & -1 \\
e^{\mathrm{i} \varphi_{n}} & e^{\mathrm{i} \varphi_{n}}
\end{array}\right)
$$

so that $\boldsymbol{c}_{n}=\mathscr{U}_{n} \tilde{\boldsymbol{c}}_{n}$. This transformation turns $H_{r}$ into

$$
H_{r}=-\sum_{n}\left(\tilde{\boldsymbol{c}}_{n}^{\dagger} \mathscr{M} e^{\mathrm{i} \theta} \tilde{\boldsymbol{c}}_{n+1}+\text { h.c. }\right) \text {. }
$$

where

$$
\mathscr{M}=e^{\mathrm{i} \pi / N}\left(\begin{array}{ll}
\left(\frac{1}{2}+\mathrm{i} \tilde{\alpha}\right) \cos \left(\frac{\pi}{N}\right) & \left(\frac{\mathrm{i}}{2}-\tilde{\alpha}\right) \sin \left(\frac{\pi}{N}\right) \\
\left(\frac{\mathrm{i}}{2}+\tilde{\alpha}\right) \sin \left(\frac{\pi}{N}\right) & \left(\frac{1}{2}-\mathrm{i} \tilde{\alpha}\right) \cos \left(\frac{\pi}{N}\right)
\end{array}\right) .
$$

Notice that the unitary transformation has eliminated the site dependence in the hopping matrix in Eq. (10). Thus, by Fourier transforming $H_{r}$ we can block-diagonalize it

$$
H_{r}=\sum_{k} \tilde{\boldsymbol{c}}_{k}^{\dagger} \tilde{\boldsymbol{h}}_{k} \tilde{\boldsymbol{c}}_{k}
$$


with

$$
\tilde{\boldsymbol{h}}_{k}=-\cos \left(\frac{\pi}{N}\right) \cos \left(k+\theta+\frac{\pi}{N}\right) \sigma_{0}+\sin \left(k+\theta+\frac{\pi}{N}\right)\left[\sin \left(\frac{\pi}{N}\right) \sigma_{x}-2 \tilde{\alpha} \sin \left(\frac{\pi}{N}\right) \sigma_{y}+2 \tilde{\alpha} \cos \left(\frac{\pi}{N}\right) \sigma_{z}\right] .
$$

In the basis that diagonalizes $\tilde{\boldsymbol{h}}_{k}$ we can write $H_{r}$ as

$$
H_{r}=\sum_{n, \mu= \pm} \varepsilon_{n \mu} d_{n \mu}^{\dagger} d_{n \mu}
$$

where the eigenvalues arep

$$
\varepsilon_{n, \pm}=-\cos \left(\frac{\pi}{N}\right) \cos \left[\frac{2 \pi}{N}\left(n+\frac{1}{2}+\Phi\right)\right] \pm \sin \left[\frac{2 \pi}{N}\left(n+\frac{1}{2}+\Phi\right)\right] \sqrt{\sin ^{2}\left(\frac{\pi}{N}\right)+4 \tilde{\alpha}^{2}} .
$$

In this basis, $H_{c}$ is written as follows

$$
H_{c}=\sum_{n, \mu= \pm}\left[\beta_{n, \mu} d_{n, \mu}^{\dagger}\left(\lambda_{-} f^{\dagger}+\lambda_{+} f\right)-\beta_{n, \mu}^{*} d_{n, \mu}\left(\lambda_{-} f+\lambda_{+} f^{\dagger}\right)\right],
$$

where $\lambda_{ \pm}=\left(\lambda_{1} \pm \lambda_{2}\right) / 2, \beta_{n,-}=e^{-\mathrm{i} 2 \pi n / N}\left(\psi_{-}^{u}-\psi_{+}^{u}\right), \beta_{n,+}=e^{-\mathrm{i} 2 \pi n / N}\left(\psi_{-}^{d}-\psi_{+}^{d}\right)$ with

$$
\begin{aligned}
& \psi_{ \pm}^{u}=u_{ \pm} / \sqrt{1+\left|u_{ \pm}\right|^{2}} \\
& \psi_{ \pm}^{d}=1 / \sqrt{1+\left|u_{ \pm}\right|^{2}}, \\
& u_{ \pm}=\frac{2 \tilde{\alpha} \cos \left(\frac{\pi}{N}\right) \pm \sqrt{\sin ^{2}\left(\frac{\pi}{N}\right)+4 \tilde{\alpha}^{2}}}{(1-2 \mathrm{i} \tilde{\alpha}) \sin \left(\frac{\pi}{N}\right)} .
\end{aligned}
$$

We can now find the spectrum of $H$ by using the Bogoliubov-de Gennes (BdG) Hamiltonian. Indeed, if we introduce

$$
\Psi=\left(\left\{d_{n, \mu}\right\}, f,\left\{d_{n, \mu}^{\dagger}\right\}, f^{\dagger}\right)^{T},
$$

with $\left\{d_{n, \mu}\right\}=d_{1,-}, d_{1,+}, \ldots, d_{N,-}, d_{N,+}$ and likewise for $\left\{d_{n, \mu}^{\dagger}\right\}$, we can write $H$ as

$$
H=\frac{1}{2} \boldsymbol{\Psi}^{\dagger} \mathscr{H}_{\mathrm{BdG}} \boldsymbol{\Psi}
$$

where

$$
\mathscr{H}_{\mathrm{BdG}}=\left(\begin{array}{cc}
\mathscr{H} & \Delta \\
-\Delta^{*} & -\mathscr{H}^{*}
\end{array}\right),
$$

is the BdG Hamiltonian. Here, $\mathscr{H}=\mathscr{H}_{0}+\mathscr{V}$ and $\Delta$ are the following $(2 N+1) \times(2 N+1)$ matrices

$$
\mathscr{H}_{0}=\operatorname{diag}\left[\varepsilon_{1,-}, \varepsilon_{1,+}, \ldots, \varepsilon_{N,-}, \varepsilon_{N,+}, \xi_{M}\right]
$$

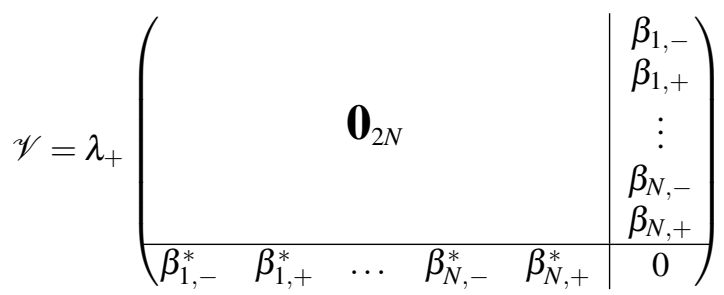

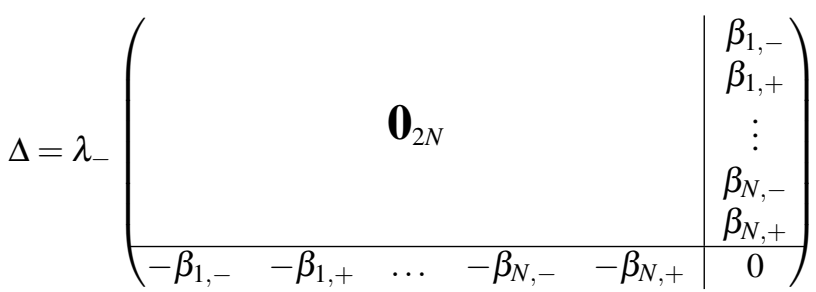

where $\mathbf{0}_{2 N}$ is the $2 N \times 2 N$ zero matrix. The fact that we can write the BdG Hamiltonian is a consequence of particle-hole symmetry and, as a result, the spectrum will be symmetrical around $E=0$. Diagonalization of $\mathscr{H}_{\mathrm{BdG}}$ provides us with the quasiparticle spectrum, $E(\Phi)$. 


\section{Quasiparticle energy spectra}

In figures $1-4$, we show the quasiparticle spectrum for rings with $N=3$ and $N=4$ in different scenarios. In figure 1 , we consider the case of $\alpha=0$ and coupling to a single Majorana mode, namely to $\gamma_{1}$. In this case, we can observe that the spectrum is symmetric as $E(\Phi)=E(-\Phi)$ and the Majorana modes remain at zero energy [see panels (a) and (b)] as long as there is no coupling between them, that is, as long as $\xi_{M}=0$. However, the coupling to $\gamma_{1}$ breaks the double degeneracy that would otherwise be present in the Dirac cone-like features of the spectra, as can be observed at zero flux. Notice that the degeneracy is lifted only for the spin-up bands, as can be understood from the coupling of the MZMs to the quantum ring in Eq. (8). Nevertheless, this coupling does not lift extra degeneracies, as we shall show when adding Rashba spin-orbit coupling. Once the coupling between MZMs is introduced [see panels (c) and (d)], we see the Majorana oscillations that naturally arise in finite-sized nanowires ${ }^{2}$. There are special points of the spectrum, namely at the Dirac points, where the hybridization between MZMs effectively vanishes.

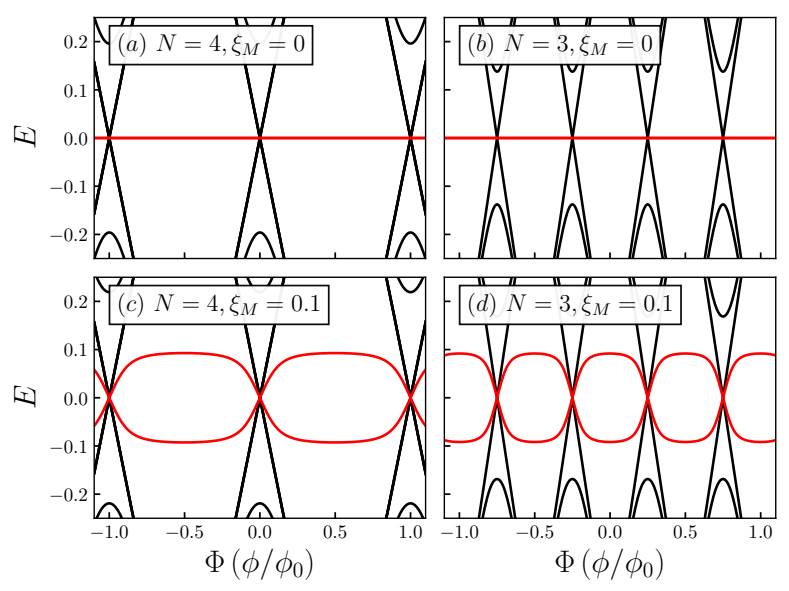

Figure 1. Quasiparticle spectra for the case of rings with $N=3$ and $N=4, \alpha=0, \lambda_{1}=0.1, \lambda_{2}=0$. Black curves represent the states in the quantum ring and the red curves correspond to the Majorana modes.

In figure 2, while the ring is still coupled to a single MZM, we introduce Rashba spin-orbit coupling. As it can be observed, the effect corresponds to a lateral shift of the bands. It can be noticed, as we anticipated above, that only certain degeneracies are lifted by the coupling to the Majorana mode. Indeed, for $N=4$ we can see that the band that shifts to the left-hand side displays both gapless and gaped Dirac-like spectra close to zero energy, while the band that moves rightwards only shows a Dirac cone-like feature. However, for $N=3$ we can see that the two shifted sides present a gaped Dirac-like spectra; this is because the Rashba spin-orbit coupling makes possible the interaction between MZMs and spin down. This shift is also observed in the oscillatory behavior of the MZMs as they are coupled together. Remarkably, however, these shifts follow the Dirac points corresponding to the spin-up states to which $\gamma_{1}$ is coupled, as we can observe in panels (c) and (d).

In figure 3 we consider the effect of coupling between the two MZMs while keeping the Rashba spin-orbit coupling turned off, $\alpha=0$. As we can observe, the degeneracy is further lifted by coupling the ring to the two MZMs, as can be observed at zero flux. However, as we expressed above, it is not entirely lifted since there are still gapless Dirac-like features at low energy. Remarkably, there is a clear distinction between even- and odd-numbered rings. Indeed, even when the MZMs are explicitly uncoupled in the Hamiltonian, when $\xi_{M}=0$, there is hybridization due to their coupling through the ring. Although we do not show it here, this behavior can be seen to occur in rings with a larger number of sites. This effect may be due to the fact that even-numbered rings with $\alpha=0$ and at zero flux present particle-hole symmetry $c_{n, \sigma} \rightarrow(-1)^{n} c_{n,-\sigma}^{\dagger}$ and $c_{n, \sigma}^{\dagger} \rightarrow(-1)^{n} c_{n,-\sigma}$, whereas odd-numbered rings do not, making these two systems inherently different. Notice that this particle-hole symmetry is different from the one found in the BdG Hamiltonian.

Finally, figure 4 shows the effect of including Rashba spin-orbit coupling, which breaks the symmetry $E(\Phi)=E(-\Phi)$ by shifting sideways the bands, as occurred in figure 2. However, in contrast to the latter, the coupling to $\gamma_{2}$ leads to a further reduction of the degeneracies in the problem. In fact, for $N=4$, the spectrum is wholly gaped out, even for $\xi_{M}=0$. There is then a clear distinction with the corresponding spectrum without Rashba spin-orbit coupling, which will affect the persistent currents, as we shall show below. 

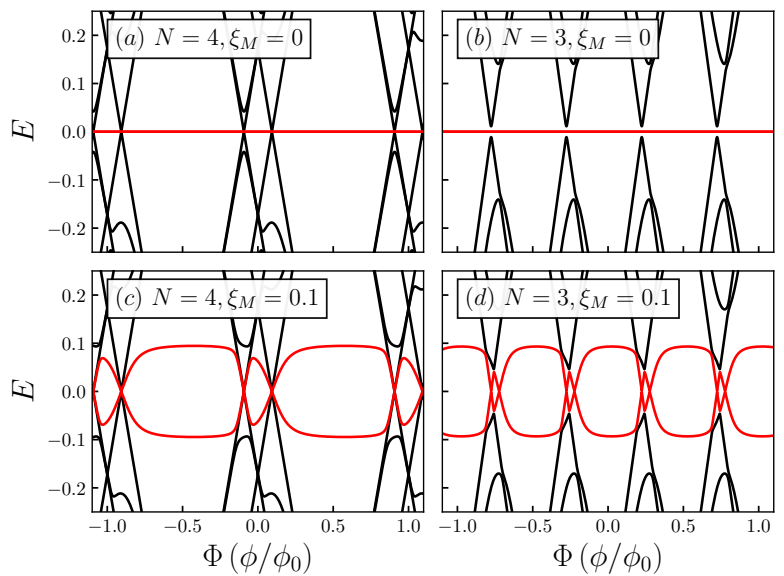

Figure 2. Quasiparticle spectra for the case of rings with $N=3$ and $N=4, \alpha=0.5, \lambda_{1}=0.1, \lambda_{2}=0$. Black curves represent the states in the quantum ring and the red curves correspond to the Majorana modes.
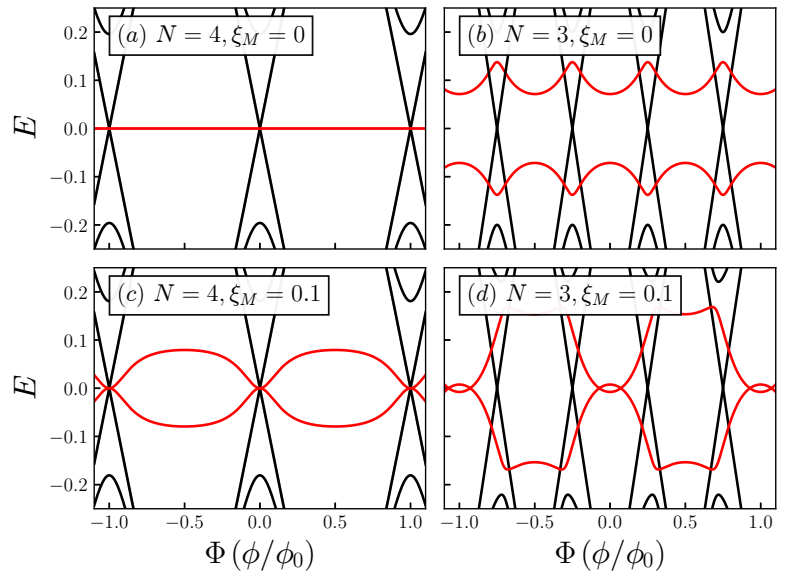

Figure 3. Quasiparticle spectra for the case of rings with $N=3$ and $N=4, \alpha=0, \lambda_{1}=0.1, \lambda_{2}=0.15$. Black curves represent the states in the quantum ring and the red curves correspond to the Majorana modes.

\section{Charge and spin persistent currents}

Persistent currents have been studied extensively, both theoretically ${ }^{15,19-24}$ and experimentally ${ }^{25-28}$. They constitute a peculiar quantum phenomenon resulting from the time-reversal symmetry breaking that occurs from coupling electrons to a gauge field, as in the Aharonov-Bohm effect.

In this section, we will study the appearance of persistent charge, $I_{q}$, and spin currents, $I_{s}$, in all the scenarios discussed above. We will calculate $I_{q}$ as

$$
I_{q}=-\mathrm{i}\left\langle\left[N_{\uparrow}+N_{\downarrow}, H_{r}\right]\right\rangle,
$$

and $I_{s}$ as

$$
I_{s}=-\mathrm{i}\left\langle\left[N_{\uparrow}-N_{\downarrow}, H_{r}\right]\right\rangle,
$$

where $N_{\sigma}=\sum_{n} c_{n, \sigma}^{\dagger} c_{n, \sigma}$. Notice that the charge persistent current can also be obtained from the eigenenergies $E_{v}$ by summing over all states $I_{q}=\sum_{v} I_{q, v}$ where ${ }^{15}$

$$
I_{q, v}=-\frac{\partial}{\partial \Phi}\left[f\left(E_{v}\right) E_{v}\right],
$$



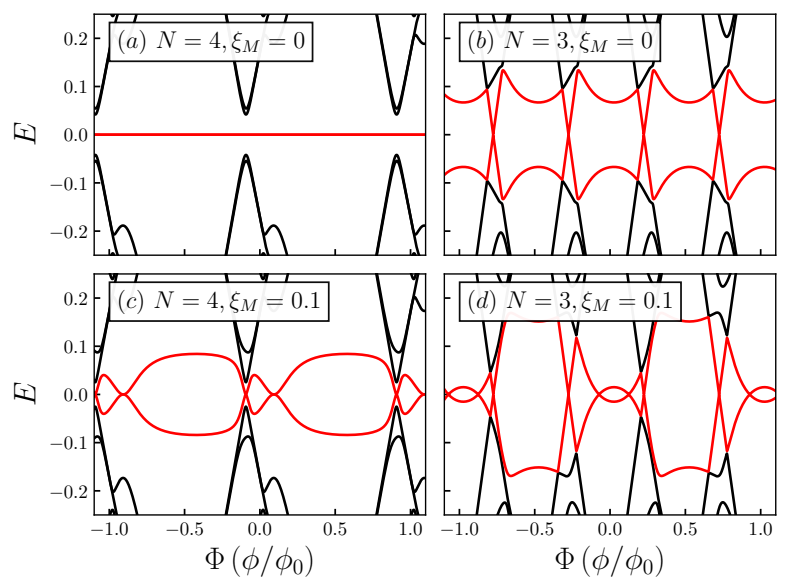

Figure 4. Quasiparticle spectra for the case of rings with $N=3$ and $N=4, \alpha=0.5, \lambda_{1}=0.1, \lambda_{2}=0.15$. Black curves represent the states in the quantum ring, and the red curves correspond to the Majorana modes.

and $f\left(E_{v}\right)$ the Fermi distribution function. The total persistent current is obtained by summing over all states ${ }^{15}$. After lengthy but straightforward calculations, we find that

$$
I_{q(s)}=\sum_{n}\left[\chi_{n}^{q(s)}+\gamma_{n}^{q(s)}-\chi_{n}^{q(s)}\left\langle d_{n,-} d_{n,-}^{\dagger}\right\rangle-\gamma_{n}^{q(s)}\left\langle d_{n,+} d_{n,+}^{\dagger}\right\rangle-2 \operatorname{Re}\left\{\beta_{n}\left\langle d_{n,+} d_{n,-}^{\dagger}\right\rangle\right\}\right] .
$$

As we can see, the expressions for the persistent currents are identical, except for the coefficients. For the charge persistent current, we find

$$
\begin{aligned}
& \chi_{n}^{q}=A_{n}^{+}\left|\psi_{-}^{u}\right|^{2}+A_{n}^{-}\left|\psi_{+}^{u}\right|^{2}+2 \operatorname{Re}\left\{B_{n} \psi_{-}^{u}\left(\psi_{+}^{u}\right)^{*}\right\}, \\
& \gamma_{n}^{q}=A_{n}^{+}\left|\psi_{-}^{d}\right|^{2}+A_{n}^{-}\left|\psi_{+}^{d}\right|^{2}+2 \operatorname{Re}\left\{B_{n} \psi_{-}^{d}\left(\psi_{+}^{d}\right)^{*}\right\}, \\
& \beta_{n}=A_{n}^{+} \psi_{-}^{u}\left(\psi_{-}^{d}\right)^{*}+A_{n}^{-} \psi_{+}^{u}\left(\psi_{+}^{d}\right)^{*}+B_{n} \psi_{-}^{u}\left(\psi_{+}^{d}\right)^{*}+B_{n}^{*} \psi_{+}^{u}\left(\psi_{-}^{d}\right)^{*},
\end{aligned}
$$

where

$$
\begin{aligned}
A_{n}^{ \pm} & =\left[\sin \left(\frac{\pi}{N}+\frac{2 \pi}{N} \Phi+\frac{2 \pi n}{N}\right) \pm 2 \tilde{\alpha} \cos \left(\frac{\pi}{N}+\frac{2 \pi}{N} \Phi+\frac{2 \pi n}{N}\right)\right] \cos \left(\frac{\pi}{N}\right), \\
B_{n} & =(1+i 2 \tilde{\alpha}) \cos \left(\frac{\pi}{N}+\frac{2 \pi}{N} \Phi+\frac{2 \pi n}{N}\right) \sin \left(\frac{\pi}{N}\right) .
\end{aligned}
$$

The expressions for the spin persistent current coefficients are identical by replacing $\sin \leftrightarrow \cos$ in $A_{n}^{ \pm}$and $B_{n}$ and by taking $\tilde{\alpha} \rightarrow-\tilde{\alpha}$ in $B_{n}$. Considering the fluctuation-dissipation theorem, we can write

$$
I_{q(s)}=\sum_{n} \frac{1}{\pi} \int_{-\infty}^{\infty} d \omega f(\omega)\left[\chi_{n}^{q(s)} \operatorname{Im}\left\langle\left\langle d_{n,-} ; d_{n,-}^{\dagger}\right\rangle\right\rangle_{\omega}+2 \operatorname{Re}\left\{\beta_{n} \operatorname{Im}\left\langle\left\langle d_{n,-} ; d_{n,+}^{\dagger}\right\rangle\right\rangle_{\omega}\right\}+\gamma_{n}^{q(s)} \operatorname{Im}\left\langle\left\langle d_{n,+} ; d_{n,+}^{\dagger}\right\rangle\right\rangle_{\omega}\right] .
$$

Here $\langle\langle\ldots\rangle\rangle$ stands for the Green's functions of the ring. Notice that we have dropped the first two terms in Eq. (27), $\chi_{n}^{q(s)}$ and $\gamma_{n}^{q(s)}$, since they end up cancelling out. The Green's functions can be obtained by applying the equation of motion technique ${ }^{29}$, which are found to be

$$
\begin{aligned}
& \left\langle\left\langle d_{n,-} ; d_{n,-}^{\dagger}\right\rangle\right\rangle=g_{p,-}+\frac{g_{p,-}^{2}\left|\beta_{n,-}\right|^{2}}{\tilde{M}_{p} \tilde{M}_{h}-\tilde{S}^{2}}\left[\lambda_{-}^{2} \tilde{M}_{p}+\lambda_{+}^{2} \tilde{M}_{h}+2 \lambda_{+} \lambda_{-} \tilde{S}\right] \\
& \left\langle\left\langle d_{n,+} ; d_{n,+}^{\dagger}\right\rangle\right\rangle=g_{p,+}+\frac{g_{p,+}^{2}\left|\beta_{n,+}\right|^{2}}{\tilde{M}_{p} \tilde{M}_{h}-\tilde{S}^{2}}\left[\lambda_{-}^{2} \tilde{M}_{p}+\lambda_{+}^{2} \tilde{M}_{h}+2 \lambda_{+} \lambda_{-} \tilde{S}\right], \\
& \left\langle\left\langle d_{n,-} ; d_{n,+}^{\dagger}\right\rangle\right\rangle=\frac{g_{p,-} \beta_{n-} g_{p,+} \beta_{n+}^{*}}{\tilde{M}_{p} \tilde{M}_{h}-\tilde{S}^{2}}\left[\lambda_{-}^{2} \tilde{M}_{p}+\lambda_{+}^{2} \tilde{M}_{h}+2 \lambda_{+} \lambda_{-} \tilde{S}\right],
\end{aligned}
$$


where $g_{p, \pm}=\left(z-\varepsilon_{n, \pm}\right)^{-1}$ are the Green's functions of the quantum ring in the absence of interaction with the MZMz and

$$
\begin{aligned}
\tilde{M}_{p} & =z-\xi_{M}-\sum_{n}\left(\lambda_{-}^{2} S_{h}+\lambda_{+}^{2} S_{p}\right), \\
\tilde{M}_{h} & =z+\xi_{M}-\sum_{n}\left(\lambda_{+}^{2} S_{h}+\lambda_{-}^{2} S_{p}\right), \\
\tilde{S} & =\lambda_{+} \lambda_{-} \sum_{n}\left(\left|\beta_{n,-}\right|^{2} g_{h,-}+\left|\beta_{n,+}\right|^{2} g_{h,+}+\left|\beta_{n,-}\right|^{2} g_{p,-}+\left|\beta_{n,+}\right|^{2} g_{p,+}\right) .
\end{aligned}
$$

Here $g_{h, \pm}=\left(z+\varepsilon_{n, \pm}\right)^{-1}$ are the Green's functions for holes of the ring uncoupled to the MZMs. In order to assess the effect of having MZMs, we first show in figure 5 the charge and spin persistent currents for the system without MZMs. Here, panels (a) and (b) correspond to the charge persistent currents for the $N=3$ and $N=4$ cases respectively and panels (c), (d) correspond to the spin persistent currents for the $N=3$ and $N=4$ cases respectively. The different colours indicate the value of the Rashba spin-orbit interaction.
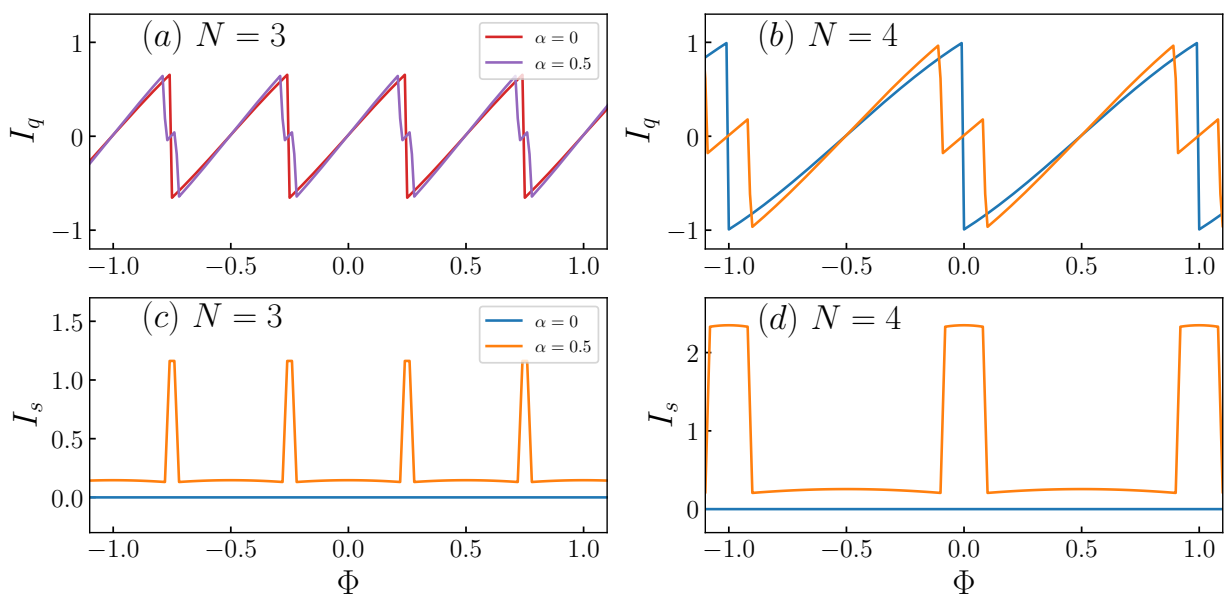

Figure 5. Charge persistent currents for (a) $N=3$ and (b) $N=4$. Spin persistent currents for (c) $N=3$ and (d) $N=4$. The parameters chosen for the two figures are $\lambda_{1}=0, \lambda_{2}=0, \xi_{M}=0$. Colours are used to indicate the value of $\alpha$.

We now study the persistent currents for the cases discussed in figures 1-4 and obtain figures 6-9. Here we use the same labeling as in the last figure, but the colours indicate three different values of $\xi_{M}$, one being $\xi_{M}=0$ [panels (a) and (b) in the BdG spectra], another being $\xi_{M}=0.1$ [panels (c) and (d) in the BdG spectra] and another one in between, $\xi_{M}=0.05$, whose spectra is not shown.

Figure 6 corresponds to the settings of figure 1. The first thing we observe is that the persistent currents display the periodicity observed in the spectra, as it should. In the case of the charge currents, we can see that upon decreasing the interaction between the two MZMs, the peaks in the current become smoother. On the other hand, the spin currents are nonzero due to the coupling of one of the two MZMs to the up-spin electrons in the ring. However, as the coupling between MZMs increases, reducing their topological robustness, the spin current tends towards zero. This is consistent with the results shown in the figure 5 as a greater interaction hybridizes the Majoranas.

Figure 7 refers to the scenario considered in figure 2, which is identical to the previous one except for a nonzero Rashba spin-orbit coupling. As we can see, in this case, the charge current is affected, as signaled by the appearance of additional peaks. Since the position of these peaks depends on the value of $\alpha$, we can fine-tune the persistent current by manipulating this parameter. However, the sharpest distinction occurs when considering the spin currents. As we can observe, Rashba renders the spin currents asymmetric around zero current and, additionally, it leads to a pulse-like pattern, similar to the one we can see in figure 5.

In figure 8 we show the persistent currents for the parameters of figure 3. In this case, the Rashba spin-orbit coupling is turned off, but the two MZMs are coupled to the ring. As we can observe, the results for the charge current are not dramatically altered, except for the curves being smoother in the $N=4$ case and for a secondary peaks at $N=3$ as $\xi_{M}$ is increased to $\xi_{M}=0.1$. These peaks feature in a clearer fashion in the spin currents, where a small plateau for $\xi_{M}=0.1$ is observed at certain values of flux. This can be understood from figure 3. Take for instance the spectrum around $\Phi=0$ in panel (d) of that figure. Although it is very subtle, there is a switching of the Majorana states at zero energy and it is in the region between 

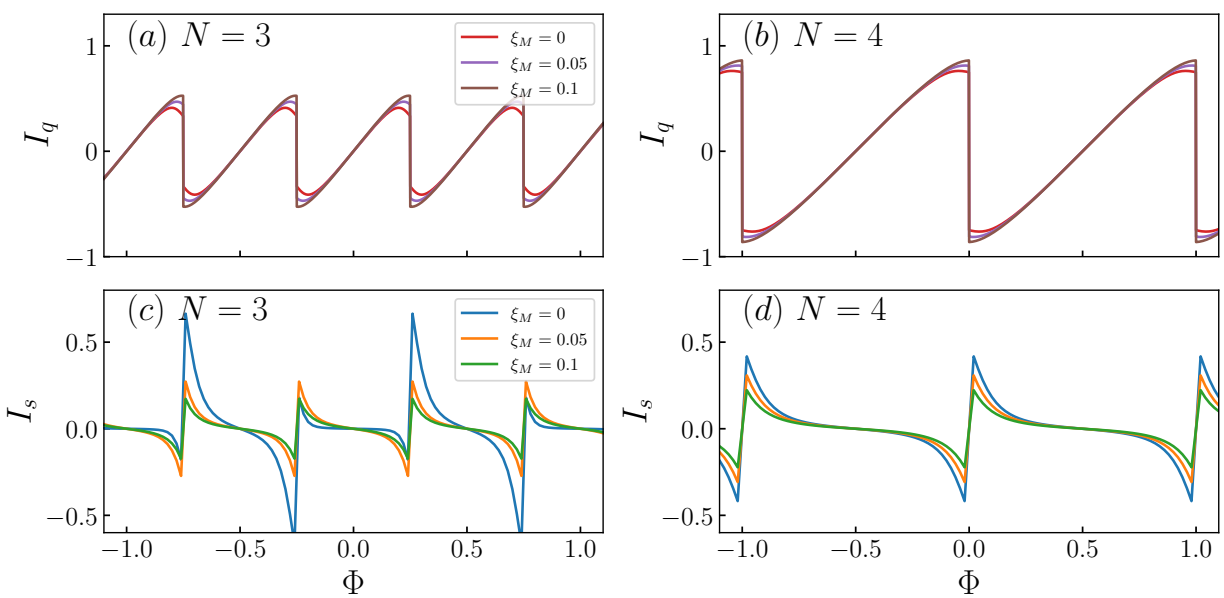

Figure 6. Charge persistent currents for (a) $N=3$ and (b) $N=4$. Spin persistent currents for (c) $N=3$ and (d) $N=4$. The parameters chosen for the two figures are $\lambda_{1}=0.1, \lambda_{2}=0, \alpha=0$. Colours are used to indicate the value of $\xi_{M}$.
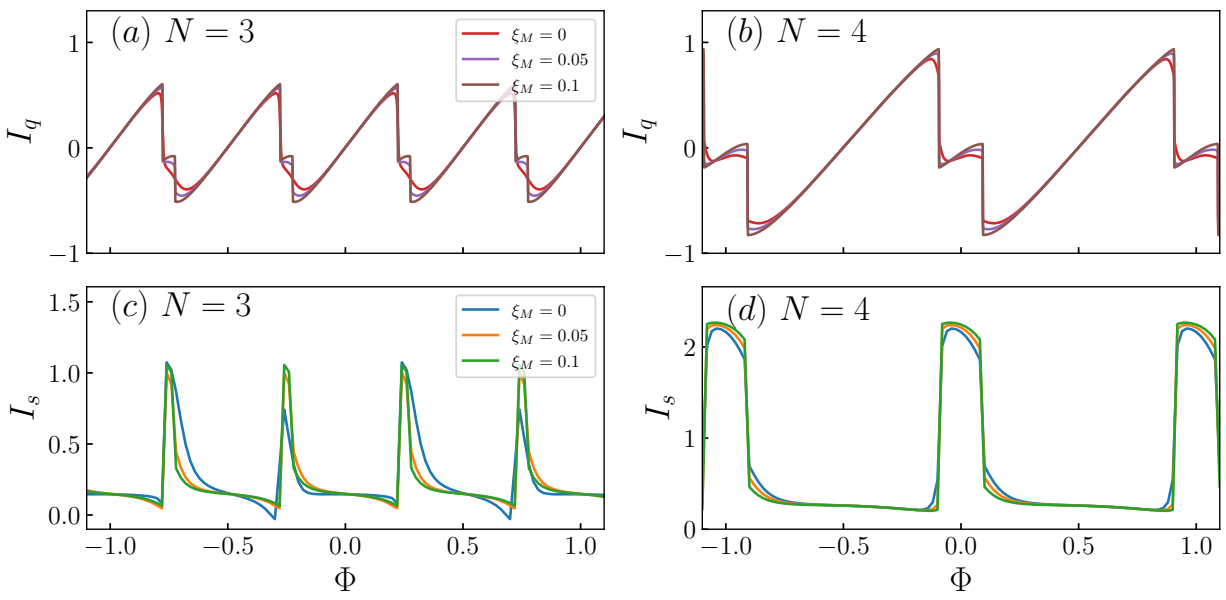

Figure 7. Charge persistent currents for (a) $N=3$ and (b) $N=4$. Spin persistent currents for (c) $N=3$ and (d) $N=4$. The parameters chosen for the two figures are $\lambda_{1}=0.1, \lambda_{2}=0, \alpha=0.5$. Colours are used to indicate the value of $\xi_{M}$.

the two crossings where this plateau takes place. Additionally, we can observe that there is no suppression of the spin current upon increasing $\xi_{M}$, as there was when $\lambda_{2}=0$. Therefore, we can see that, by coupling the ring to the two MZMs we are able to suppress the spin current for a range of fluxes. This could be a smoking gun in the detection of MZMs as this situation is completely different from the result in the absence of MZMs (cf. figure 5).

Finally, figure 9 corresponds to the case shown in figure 4. In this scenario, the currents are similar to those of figure 8, but with the additional peak that was observed for $N=4$ in figure 7 (b), although clearly smoothed out. In fact, the additional peak for the $N=3$ case is completely suppressed. This has an effect on the spin current, where the $N=4$ case still shows a pulse-like behaviour, but the $N=3$ case shows plateaus for a larger range of fluxes than in figure 8 .

\section{Conclusions}

MZMs stand out for their remarkable properties in the realm of topological quantum matter, such as their non-Abelian statistics. However, although significant progress has been made in the direction of their experimental detection in recent years, they cannot be confirmed as yet. More importantly, recent discoveries show that it is necessary to carefully examine different effects to determine their existence ${ }^{30}$ unambiguously. In this regard, we propose to consider a topological superconducting nanowire in proximity to a quantum ring threaded by a magnetic flux. It is the natural extension of the system presented in Ref. ${ }^{17}$ where a nanowire is coupled to a single quantum dot. In addition, we propose the ring to display Rashba spin-orbit coupling. As we 

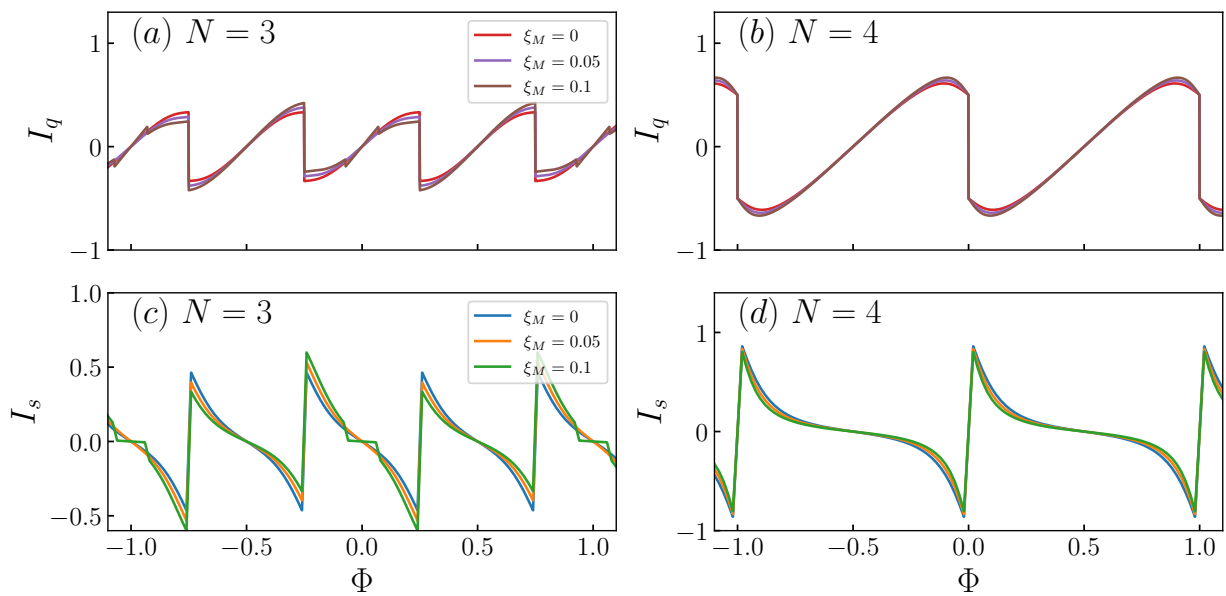

Figure 8. Charge persistent currents for (a) $N=3$ and (b) $N=4$. Spin persistent currents for (c) $N=3$ and (d) $N=4$. The parameters chosen for the two figures are $\lambda_{1}=0.1, \lambda_{2}=0.15, \alpha=0$. Colours are used to indicate the value of $\xi_{M}$.
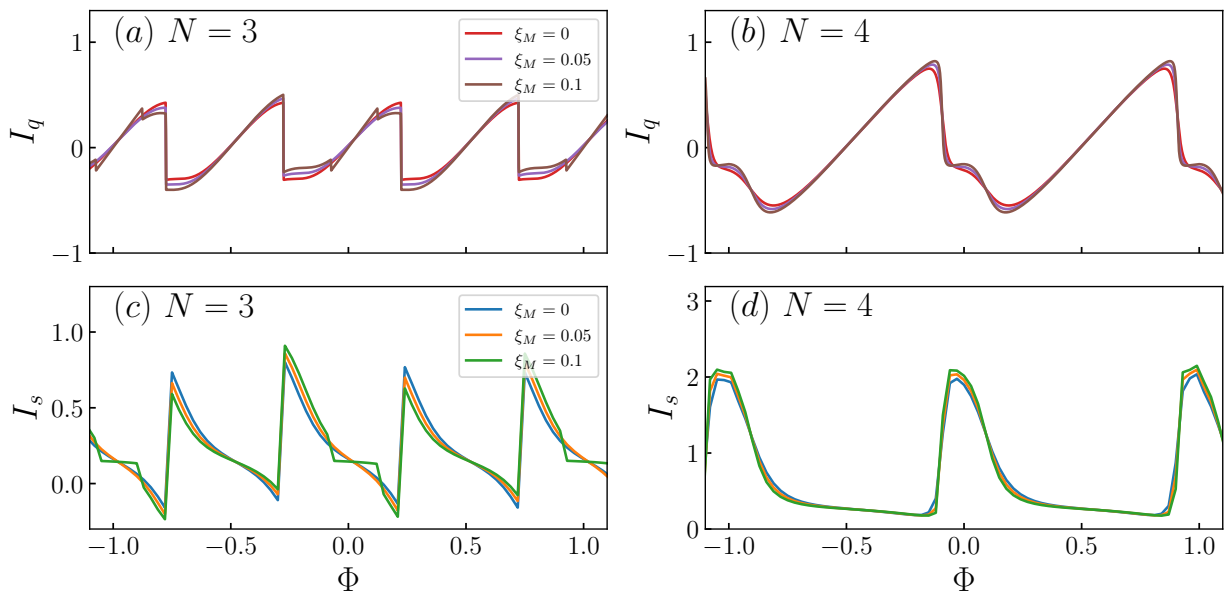

Figure 9. Charge persistent currents for (a) $N=3$ and (b) $N=4$. The parameters chosen for the two figures are $\lambda_{1}=0.1, \lambda_{2}=0.15, \alpha=0.5$. Colours are used to indicate the value of $\xi_{M}$. Spin persistent currents for (a) $N=3$ and (b) $N=4$. The parameters chosen for the two figures are $\lambda_{1}=0.1, \lambda_{2}=0.15, \alpha=0.5$. Colours are used to indicate the value of $\xi_{M}$.

have shown in this paper, the BdG spectra are entirely altered by coupling the quantum ring to one or two MZMs and having a nonzero Rashba spin-orbit coupling. The consequences are clearly observed in the persistent currents. Remarkably, spin-orbit coupling leads to an asymmetric spin current with a square pulse-like behavior. Moreover, the coupling to the two MZMs leads to plateaus for odd-numbered rings due to the switching of MZMs at zero energy. We believe that our work could serve as an additional signature towards probing the existence of Majorana zero modes.

\section{References}

1. Beenakker, C. Search for majorana fermions in superconductors. Annu. Rev. Condens. Matter Phys. 4, 113-136 (2013).

2. Aguado, R. Majorana quasiparticles in condensed matter. La Rivista del Nuovo Cimento 40, 523-593 (2017).

3. Stanescu, T. D. \& Tewari, S. Majorana fermions in semiconductor nanowires: fundamentals, modeling, and experiment. $J$. Physics: Condens. Matter 25, 233201 (2013).

4. Lutchyn, R. M. et al. Majorana zero modes in superconductor-semiconductor heterostructures. Nat. Rev. Mater. 3, 52 (2018). 
5. Sarma, S. D., Freedman, M. \& Nayak, C. Majorana zero modes and topological quantum computation. npj Quantum Inf. 1, $15001(2015)$.

6. Sato, M. \& Fujimoto, S. Majorana fermions and topology in superconductors. J. Phys. Soc. Jpn. 85, 072001 (2016).

7. Kitaev, A. Y. Unpaired majorana fermions in quantum wires. Physics-Uspekhi 44, 131 (2001).

8. Alicea, J. New directions in the pursuit of majorana fermions in solid state systems. Reports on Prog. Phys. 75, 076501 (2012).

9. Elliott, S. R. \& Franz, M. Colloquium: Majorana fermions in nuclear, particle, and solid-state physics. REview Mod. Phys. 87, 137 (2015).

10. Lutchyn, R. M., Sau, J. D. \& Das Sarma, S. Majorana fermions and a topological phase transition in semiconductorsuperconductor heterostructures. Phys. Rev. Lett. 105, 077001 (2010).

11. Oreg, Y., Refael, G. \& von Oppen, F. Helical liquids and majorana bound states in quantum wires. Phys. Rev. Lett. 105, 177002 (2010).

12. Deng, M. T. et al. Majorana bound state in a coupled quantum-dot hybrid-nanowire system. Science 354, 1557 (2016).

13. Liu, X., Borunda, M. F., Liu, X.-J. \& Sinova, J. Control of josephson current by aharonov-casher phase in a rashba ring. Phys. Rev. B 80, 174524 (2009).

14. Maiti, S. K., Dey, M., Sil, S., Chakrabarti, A. \& Karmakar, S. N. Magneto-transport in a mesoscopic ring with rashba and dresselhaus spin-orbit interactions. EPL (Europhysics Lett. 95, 57008 (2011).

15. Monisha, P. J., Sankar, I. V., Sil, S. \& Chatterjee, A. Persistent current in a correlated quantum ring with electron-phonon interaction in the presence of rashba interaction and aharonov-bohm flux. Sci. Reports 6, 20056 (2016).

16. Zeng, Q.-B., Chen, S., You, L. \& Lü, R. Transport through a quantum dot coupled to two majorana bound states. Front. Phys. 12, 127302 (2017).

17. Prada, E., Aguado, R. \& San-Jose, P. Measuring majorana nonlocality and spin structure with a quantum dot. Phys. Rev. B 96, 085418 (2017).

18. Ramos-Andrade, J. P., Orellana, P. A. \& Ulloa, S. E. Detecting coupling of majorana bound states with an aharonov-bohm interferometer. J. Physics: Condens. Matter 30, 045301 (2018).

19. Byers, N. \& Yang, C. N. Theoretical considerations concerning quantized magnetic flux in superconducting cylinders. Phys. Rev. Lett. 7, 46 (1961).

20. Büttiker, M., Imry, Y. \& Landauer, R. Josephson behavior in small normal one-dimensional rings. Phys. Lett. A 96, 365 (1983).

21. Ambegaokar, V. \& Eckern, U. Coherence and persistent currents in mesoscopic rings. Phys. Rev. Lett. 65, 381 (1990).

22. Riedel, E. K. \& von Oppen, F. Mesoscopic persistent current in small rings. Phys. Rev. B 47, 15449 (1993).

23. Orellana, P. A. \& Pacheco, M. Persistent current magnification in a double quantum-ring system. Phys. Rev. B 71, 235330 (2005).

24. Maiti, S. K. \& Chakrabarti, A. Magnetic response of interacting electrons in a fractal network: A mean-field approach. Phys. Rev. B 82, 184201 (2010).

25. Mailly, D., Chapelier, C. \& Benoit, A. Experimental observation of persistent currents in gaas-algaas single loop. Phys. Rev. Lett. 70, 2020 (1993).

26. Jariwala, E. M. Q., Mohanty, P., Ketchen, M. B. \& Webb, R. A. Diamagnetic persistent current in diffusive normal-metal rings. Phys. Rev. Lett. 86, 1594 (2001).

27. Bluhm, H., Koshnick, N. C., Bert, J. A., Huber, M. E. \& Moler, K. A. Persistent currents in normal metal rings. Phys. Rev. Lett. 102, 136802 (2009).

28. Bleszynski-Jayich, A. C. et al. Persistent currents in normal metal rings. Science 326, 272 (2009).

29. Jishi, R. A. Feynman Diagram Techniques in Condensed Matter Physics (Cambridge University Press, 2013).

30. Frolov, S. Quantum computing's reproducibility crisis: Majorana fermions. Nature 592, 350 (2021). 


\section{Acknowledgements}

F. G. M. is grateful for the funding of scholarship CONICYT-Chile No. 21170550. L. R. and P. A. O. acknowledges support from FONDECYT, Grants 1180914 and 1201876. Work at Madrid has been supported by Agencia Estatal de Investigación, Grant PID2019-106820RB-C21.

\section{Author contributions statement}

P. A. O., F. D. A. and L. R. conceived the research. F. G. M. and D. M. performed the calculations. A. D. F. carried out calculations and wrote the manuscript. All authors discussed the results and reviewed the manuscript. 